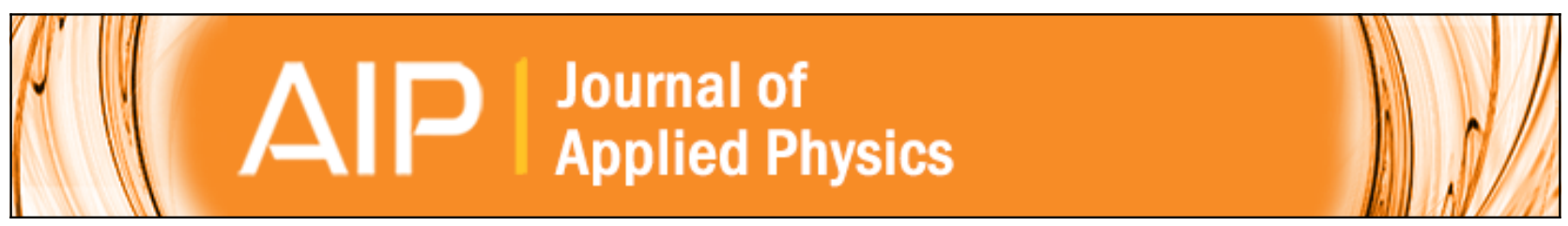

\title{
X-ray diffraction approach to grain boundary and volume diffusion
}

J. Unnam, J. A. Carpenter, and C. R. Houska

Citation: Journal of Applied Physics 44, 1957 (1973); doi: 10.1063/1.1662500

View online: http://dx.doi.org/10.1063/1.1662500

View Table of Contents: http://scitation.aip.org/content/aip/journal/jap/44/5?ver=pdfcov

Published by the AIP Publishing

\section{Articles you may be interested in}

Intrinsic stress in ZrN thin films: Evaluation of grain boundary contribution from in situ wafer curvature and ex situ $\mathrm{x}$-ray diffraction techniques

J. Appl. Phys. 111, 093509 (2012); 10.1063/1.4710530

Grain boundary transport in x-ray irradiated polycrystalline diamond

J. Appl. Phys. 93, 6078 (2003); 10.1063/1.1565191

Glancing angle x-ray diffraction: A different approach

Appl. Phys. Lett. 64, 1585 (1994); 10.1063/1.111847

X-Ray Diffraction

Phys. Today 15, 96 (1962); 10.1063/1.3057841

A Means for Eliminating "Paper Grain" from X-Ray Diffraction Films

Rev. Sci. Instrum. 18, 453 (1947); 10.1063/1.1740976

\section{MIT LINCOLN} LABORATORY CAREERS

\section{Discover the satisfaction of} innovation and service to the nation
- Space Control

- Air \& Missile Defense

- Communications Systems \& Cyber Security

Intelligence, Surveillance and

Reconnaissance Systems

- Advanced
Electronics
- Tactical Systems
- Homeland
Protection
- Air Traffic Control

MASSACHUSETTS INSTITUTE OF TECHNOLOGY

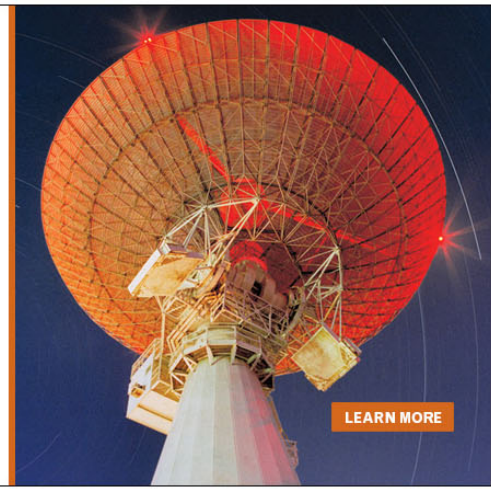




\title{
X-ray diffraction approach to grain boundary and volume diffusion
}

\author{
J. Unnam, J. A. Carpenter*, and C. R. Houska \\ Virginia Polytechnic Institute and State University, Blacksburg, Virginia 24061 \\ (Received 10 November 1972; in final form 22 January 1973)
}

A generalized two-dimensional diffusion model has been developed which consists of an array of boundaries coupled to the free surface and to the substrate lattice. The model makes use of three nonlinear partial differential equations which describe lattice, grain boundary, and surface diffusion. This two-dimensional model has been programmed for the IBM 360 computer using a finite-difference solution to give concentrations as a function of time. An x-ray intensity simulation program is developed to give integrated diffracted intensity for a given concentration distribution. This simulated intensity is compared with experimental intensity. Data are presented from a sample containing $8 \mu$ of $\mathrm{Ni}$ on a (111)-oriented $\mathrm{Cu}$ crystal diffused for various times at $900^{\circ} \mathrm{C}$ and a similar sample with $6.5 \mu$ of $\mathrm{Ni}$ diffused at $600^{\circ} \mathrm{C}$. The simulations are in good agreement with experimental intensity bands. Activation energies and frequency factors are given for volume and grain boundary diffusion which are in good agreement with those literature values that are available. After a diffusion treatment at $600{ }^{\circ} \mathrm{C}$, it was found that pipe diffusion makes an important contribution to the volume diffusion coefficient. At $900^{\circ} \mathrm{C}$ this does not appear to be true. The contribution from pipe diffusion correlates with rocking curve data except for compositions close to that of the free surface.

\section{INTRODUCTION}

In previous papers, ${ }^{1-3}$ a new $x$-ray technique has been used to study pure volume diffusion; however, highdiffusivity paths such as grain boundaries and dislocations were not directly considered. These mechanisms are known to be important at the lower and intermediate temperatures and are of significance in determining the properties of materials. The primary goal of this paper is to extend the $x$-ray method and to provide meaningful data at intermediate temperatures.

The various high-diffusivity paths associated with a generalized diffusion model may be specified in terms of their dimensionality. A boundary between two grains is a two-dimensional surface; three grains intersect to form a one-dimensional line; and the junction between four grains is a point with zero dimensionality. The dislocation, like the junction between three grains, can be treated as a one-dimensional line. Atoms may be transported along a system of interconnecting paths containing surfaces, lines, and points which may take on special orientations in space. Generally, there is a sufficiently large difference in the density of line defects to distinguish "boundary material" from "volume material". This obvious geometrical distinction has provided a basis for diffusion models. However, much of the theoretical work has treated the grain boundary diffusion problem under rather restrictive conditions.

A unified model described in later sections deals primarily with grain boundary and volume diffusion in which boundaries form a regular network and make a diffusion circuit with the free surface. It is assumed that twodimensional grain boundaries contain most of the active sites associated with the various high-diffusitivity paths, that any dislocations extending through the lattice represent a small fraction of the total number of specimen sites, and volume diffusion is three-dimensional and homogeneous. ${ }^{4}$ These conditions are most likely to be found at temperatures ranging from the melting point to roughly one-half this value. A realistic experimental approach to the diffusion problem should include structural measurements which characterize the arrangement of dislocations along diffusion zones. In this connection, an x-ray diffraction approach is capable of providing most of the required information.

The first and simplest approach to grain boundary diffusion was carried out by Fisher ${ }^{5}$ His final analytic form is sufficiently simple to apply to certain experimental data and to readily evaluate the solution under various conditions. In an attempt to apply a modified version of this form, it became evident that this solution is too restrictive to be applied to a system of boundaries within a finite coating. Also, most of the features observed in the $x$-ray intensity data could not be described using modifications of the final form. ${ }^{6}$ Other solutions ${ }^{7,8}$ were examined and were also found to be too restrictive to be applied to the study of thin coatings.

The model described here represents an extension of one treated by Suzuoka. ${ }^{9}$ Suzuoka deviated from the usual fixed boundary conditions and considered an instantaneous source which was permitted to vary in concentration with time. He found that a single grain boundary may both supply solute atoms to the lattice (source) and in another location remove them, as if it were acting as a sink. This model could not be used because of restrictive conditions; therefore, several steps were taken to obtain more realistic solutions. These include the following: (a) Lattice and grain boundary diffusion equations were generalized to allow a variation of diffusion coefficients with concentration. (b) A network of interacting boundaries were considered which provide connecting high-diffusivity circuits having a component perpendicular to the original interface. (c) The circuit between the network and free surface was also considered. These considerations, as well as an x-ray diffraction approach, are described and applied to provide an experimental example.

\section{X-RAY DIFFRACTION-HIGH-DIFFUSIVITY PATHS}

The single-crystal $x$-ray diffraction equations previously used to study volume diffusion may be applied to the present problem with certain modifications. In the case 


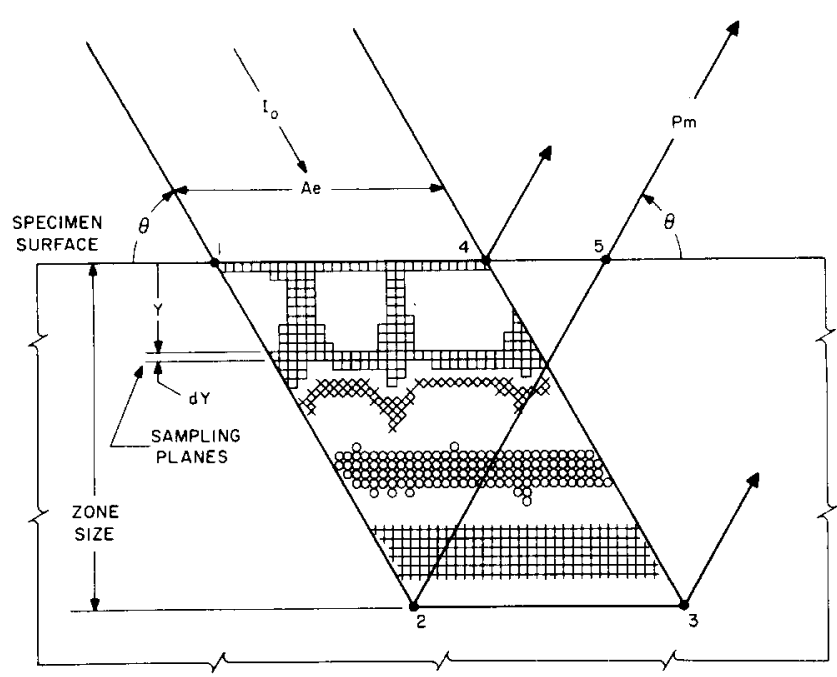

FIG. 1. Schematic cross section of a diffused specimen illustrating the irradiated region $(1-2-3-4)$ due to an $x-r a y$ beam with incident intensity $I_{0}$. Each sampling plane has a cross-sectional area $A_{e}$ which is relatable to the cross section of the incident beam $A_{0}$ and the Bragg angle $\theta$. Plane 2-3 indicates a practical lower limit of sampled region. Selected compositions are designated within the sampled zone.

of unidirectional volume diffusion at a given instant of time, there is no change in the average composition along points on a plane parallel to the original interface. Two closely spaced planes, with lattice parameters ranging from $a_{m}-\frac{1}{2} \Delta a$ to $a_{m}+\frac{1}{2} \Delta a$, can be assumed to contain material of constant composition $m$. At lower diffusion temperatures, two planes marking the same extremities would extend along the various high-diffusivity paths and form a three-dimensional cellular network of average composition $m$. The size and shape depend upon the geometry and type of imperfections within the crystalline material, the composition, the diffusion time, and temperature. Since the $x$-ray absorption factor depends upon the path length and composition of material above a diffracting element, this term is more difficult to treat exactly than the comparable factor for volume diffusion.

If the cross-sectional area of the incident beam is $A_{0}$, the area of specimen sampled by the $x$-ray beam is given by $A_{e}=A_{0} / \sin \theta$ (see Fig. 1). A sampled region ranges from the surface plane 1-4 to a plane 2-3. Regions below plane 2-3 cannot provide diffracted intensity in excess of the statistical fluctuations. In general, the location of the limiting plane 2-3 will depend upon $\theta$ as well as the linear absorption coefficient for path 1-2-5. As a sampling plane with cross section $A_{e}$ is lowered below the surface to increasing depths, it can be imagined to intersect irregular composition surfaces resulting from high-diffusivity paths. The fraction of a rea of composition $m$ intersected by the sampling plane at depth $Y$ is defined by the function $H_{m}(Y)$. This is related to the total volume of material of this composition. Since the $x$-ray intensity is proportional to the volume of diffracting material with average composition $m$, the quantity $H_{m}(Y)$ will be of significance in later calculations. If $H_{m}(Y)$ is integrated over its full range, one obtains the total volume of composition $m$ or

$$
V_{m}=A_{e} \int_{0}^{\infty} H_{m}(Y) d Y
$$

Equation (1) cannot be used directly in the diffraction expression since it neglects absorption of the incident and diffracted beams, i.e., the term

$$
\exp \left[-k_{m} \overline{\mu(Y)} Y\right]
$$

with

$$
k_{m}=2 / \sin \theta_{m},
$$

must be included. The angle $\theta_{m}$ is the Bragg angle for element $m$, and $\overline{\mu(Y)}$ is the average linear absorption coefficient for all material from the surface to a depth $Y$. It is convenient to define an absorption-weighted volume element given by

$$
V_{m e}^{g}=g A_{e} \int_{0}^{\infty} H_{m}(Y) \exp \left[-k_{m} \overline{\mu(Y)} Y\right] d Y,
$$

where $V_{m e}^{g}$ is the effective volume. The factor $g$ is introduced to allow for large misorientations in the plating which are relatable to the annealing texture. This term represents the fraction of grains in the region originally occupied by the plating with approximately the same orientation as the substrate crystal (within about $\pm 4^{\circ}$ ).

For a given band, one would obtain the same diffracted intensity from material of composition $m$ if the equivalent volume $V_{m e}^{g}$ were located at the free surface. Because of the angular dependence contained in the absorption term and $A_{e}$, this will vary from band to band. The diffracted intensity is proportional to the effective volume $V_{m e}^{g}$ and is given in terms of the following twocrystal kinematic expression:

$$
P_{m}=I_{0} Q_{m} V_{m e}^{g},
$$

where

$$
Q_{m}=\frac{r_{e}^{2} \lambda^{3}}{v_{m}^{2}} \frac{1+\cos ^{2}(2 \alpha) \cos ^{2}\left(2 \theta_{m}\right)}{\sin \left(2 \theta_{m}\right)\left[1+\cos ^{2}(2 \alpha)\right]} F_{m}^{2} \exp \left(-2 M_{m}\right),
$$

and the constant terms are $I_{0}$-intensity of incident beam, $r_{e}$-classical radius of the electron, and $\lambda-\mathrm{x}-$ ray wavelength. The following terms depend upon the average composition $m$ of the diffracting element: $v_{m}-$

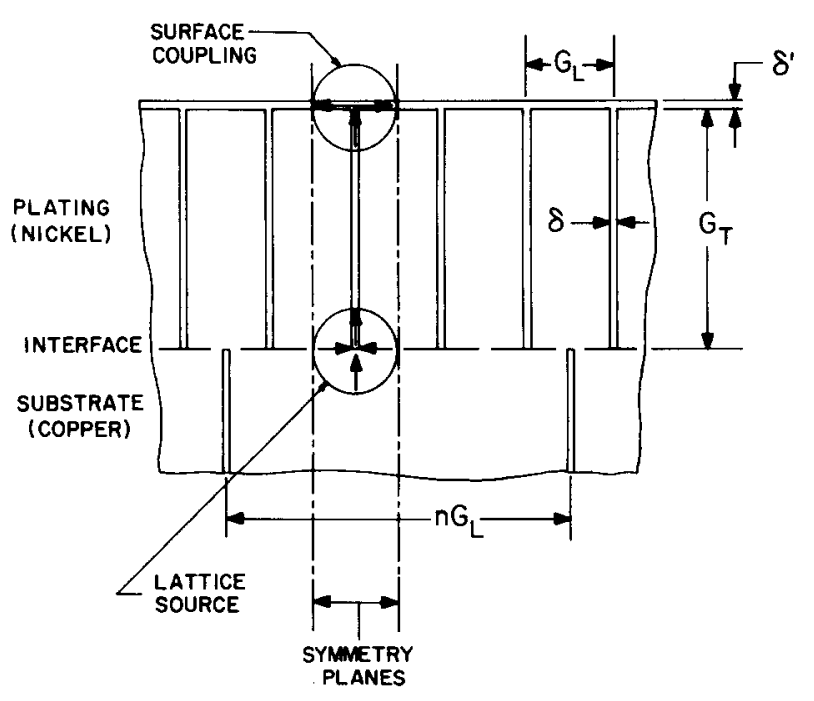

FIG. 2. Two-dimensional arrays of regularly spaced isotropic planar boundaries with spacings $G_{L}$ and $n G_{L}$. Symmetry planes are designated only for the plating. Atomic currents between the substrate and upper boundaries are coupled at lattice source points while boundary currents reaching the free surface split equally into two currents at coupling points. 


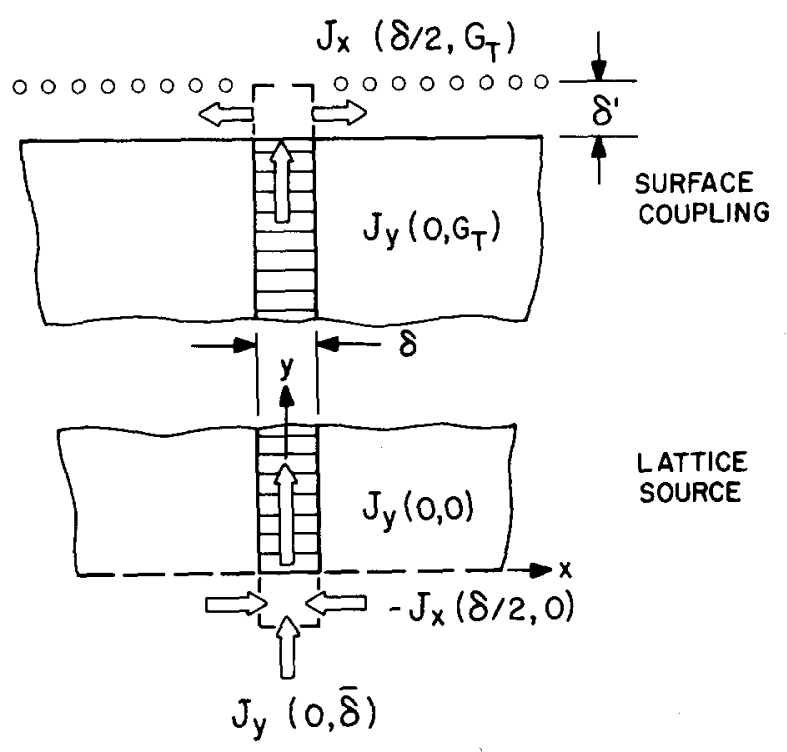

FIG. 3. Schematic representation of lattice source and surface coupling points. The grain boundary width $\delta$ and the comparable surface width $\delta^{\prime}$ are illustrated along with the various fluxes used in the circuit equations.

volume of unit cell, $F_{m}-$ structure factor, $\exp \left(-2 M_{m}\right)-$ Debye Waller factor, and $\theta_{m}, \alpha-$ Bragg angle of specimen and monochromator.

In order to proceed with the interpretation of grain boundary diffusion experiments, it is necessary to develop a diffusion model giving the variation of effective volume with composition. This contains the various diffusion coefficients as well as certain structural information.

\section{GRAIN BOUNDARY DIFFUSION MODEL}

The $\mathrm{x}$-ray technique described in the previous section is capable of giving diffusion information on films ranging in thickness from about half a micron to hundreds of microns. A typical film thickness is given by $1 / 2 \bar{\mu}$. Therefore, the thickness will depend upon the $x$-ray wavelength and the specific elements used in the investi- gation. For example, if a Au coating is to be studied with $\mathrm{Mo} K_{\alpha}$ radiation, a typical thickness should be about one micron. For a $\mathrm{Ni}$ electrodeposit onto a $\mathrm{Cu}$ substrate, a thickness of 6-8 $\mu$ gives good results with $\mathrm{Cu} K_{\alpha}$ radiation. Prior experimental results have demonstrated that a $\mathrm{Cu}$ single-crystal substrate can be obtained with much fewer boundaries per unit length than the electrodeposit, and a subsequent diffusion anneal generally alters the texture of the plating. This has been found to occur largely before measurable diffusion takes place. Our findings are compatible with a columnar structure formed initially by electrodeposition or by subsequent growth. Like prior treatments, ${ }^{5,7,9}$ the following represents a two-dimensional approximation to the three-dimensional arrangement of columns. A threedimensional model, although more representative of the actual structure, would require additional parameters and excessive computer time.

There is a sufficient body of literature available to clearly indicate that many simplifying assumptions are required in order to obtain analytical solutions. The primary goal here is to develop a diffusion model which is capable of giving simulations in agreement with the experimentally measured $\mathrm{x}$-ray intensity bands. Introducing a composition dependence into these diffusion coefficients rules out the possibility of finding usable analytical solutions to the system of partial differential equations which describe grain boundary diffusion. Consequently, only numerical solutions are considered and further restricted to the case of a single-phase binary system.

As previously indicated, the electroplating will be represented by a two-dimensional array of regularly spaced isotropic planar boundaries of thickness $\delta$ which are perpendicular to the free surface. This is illustrated in Figs. 2 and 3 . The spacing $G_{L}$ is taken to be an average distance between boundaries, $G_{T}$ is the plating thickness, and $n G_{L}$ is the average distance between boundaries in the substrate. Since the substrate is taken to be a single crystal of moderate perfection, it may be assumed that $n \gg 1$. Because of the large spacing between

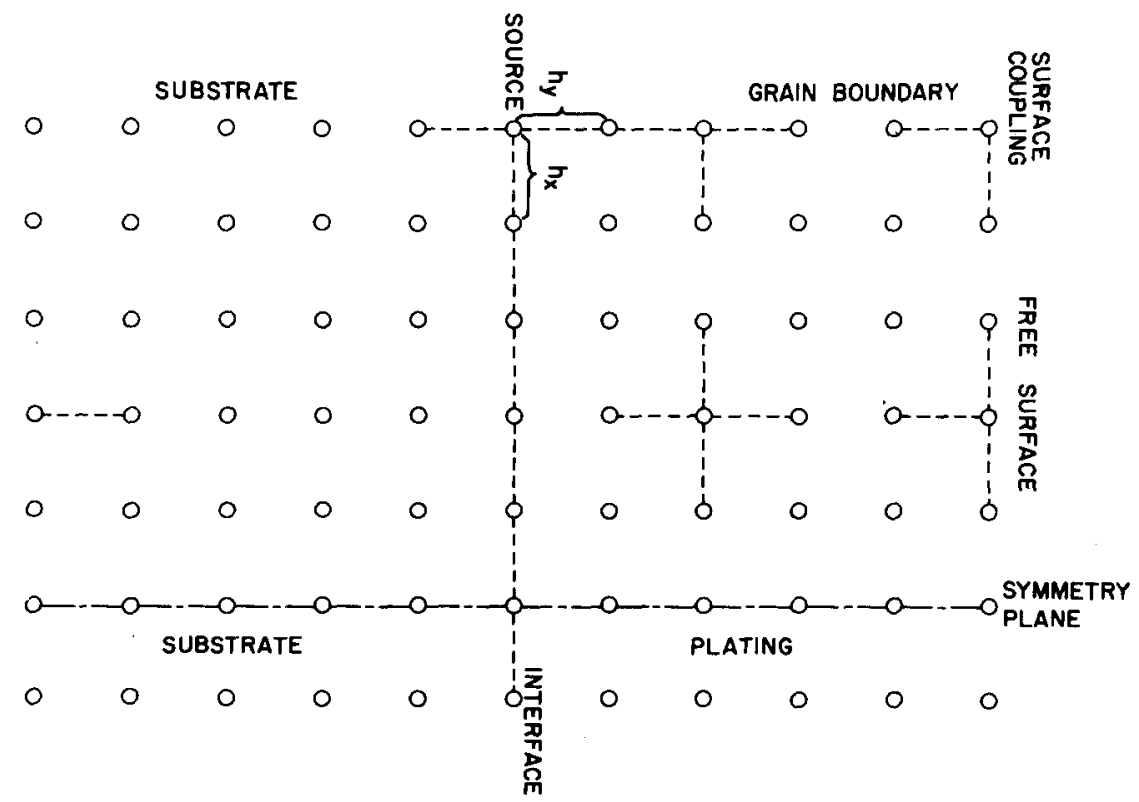

FIG. 4. Two-dimensional finite-difference network illustrating the five points used for volume calculations as well as the four points used for boundary and surface calculations. Three points are illustrated for a source and surface coupling. 
boundaries within the $\mathrm{Cu}$, these will be temporarily neglected and attention should be focused on the plated material. The plating is taken to be of finite size relative to diffusion distances and the substrate is infinite. This array of boundaries is connected by high-diffusivity paths along the surface of width $\delta^{\prime}$. For each pair of boundaries, a plane of symmetry is constructed midway between each pair; consequently, a zero-slope boundary condition is imposed on the concentration along the $x$ direction ( $x$ and $y$ coordinates are illustrated in Fig. 3).

The problem requires solutions to three nonlinear partial differential equations. If a region of material is considered away from a boundary or free surface, the equation takes the well-known two-dimensional form of Fick's second law in terms of normalized concentration $C$, time $t$, and the coordinates $x$ and $y$ :

$$
\frac{\partial C}{\partial t}=\frac{\partial}{\partial x}\left(D(C) \frac{\partial C}{\partial x}\right)+\frac{\partial}{\partial y}\left(D(C) \frac{\partial C}{\partial y}\right)
$$

This also describes points along the original interface but excludes source coupling points. By taking an element within the grain boundary and allowing the grain boundary diffusion coefficient to vary with composition in the same way as the lattice diffusion coefficient, one obtains

$$
\frac{\partial C}{\partial t}=\mathscr{D}_{b} \frac{\partial}{\partial y}\left(D(C) \frac{\partial C}{\partial y}\right)+\frac{2}{\delta}\left(D(C) \frac{\partial C}{\partial x}\right)
$$

The grain boundary diffusion coefficient has been taken to be

$$
D_{b}=\mathscr{D}_{b} D(C),
$$

where $\mathscr{D}_{b}$ is independent of composition but temperature dependent. Further details concerning the form of (8) are given in the Appendix. Equation (7) is similar to Fisher's $s^{5} \mathrm{Eq}$. (3) but expressed in a nonlinear form. The differential equation for diffusion at the free surface is

$$
\frac{\partial C}{\partial t}=\mathscr{D}_{s} \frac{\partial}{\partial x}\left(D(C) \frac{\partial C}{\partial x}\right)-\frac{1}{\delta^{\prime}}\left(D(C) \frac{\partial C}{\partial y}\right)
$$

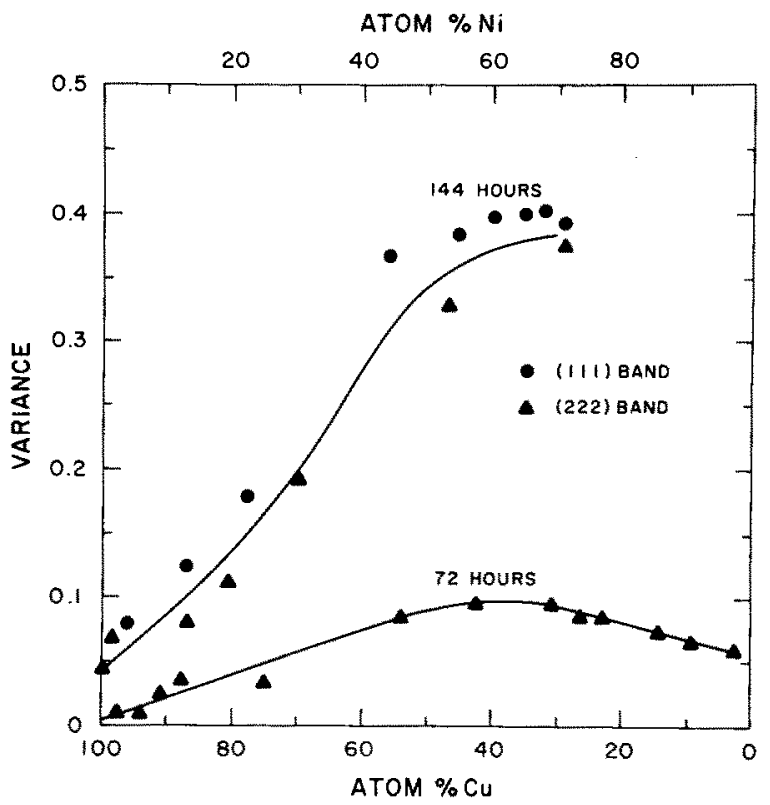

FIG. 5. Variance curves for crystal misorientation resulting from diffusion at $600^{\circ} \mathrm{C}$ after 72 and $144 \mathrm{~h}$.

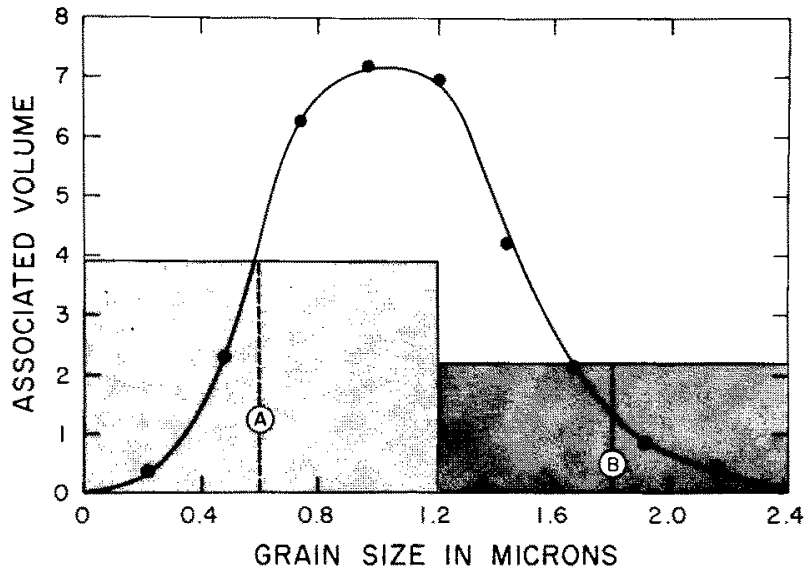

FIG. 6. Distribution of specimen volume according to grain size for a specimen diffused at $600^{\circ} \mathrm{C}$ for $144 \mathrm{~h}$ (solid curve and points). Grains $A$ and $B$ represent two grain sizes $(0.6$ and $1.8 \mu$ ) used in the simulation.

and likewise the surface diffusivity is assumed to be

$$
D_{s}=\mathscr{D}_{s} D(C) \text {. }
$$

Equation (9) was also considered by Fisher in its linear form. The second term in (9) differs by a factor of $\frac{1}{2}$ from (7) because atoms are not permitted to escape from the free surface, whereas atoms may leak out of the grain boundary from two directions. Also, the minus sign appears because back diffusion from the free surface is in the $-y$ direction.

Equations (7) and (9) represent a system of paths which include the grain boundaries and connecting surface paths. In each case, a portion of the atomic boundary and surface flux may leak into the lattice. If $\mathrm{Cu}$ diffusion into the $\mathrm{Ni}$ is being considered, the source is located within the substrate. Initially, the flux feeding the Ni boundary may be considered to pass through a cross section of $\delta \times 1 \mathrm{~cm}^{2}$. Two flux vectors in the $\pm x$ directions as well as one in the $+y$ direction must be summed to determine the net flux flowing into the boundary (see Fig. 3). It is assumed that all of the atomic current flowing into an imaginary box of cross section $\delta \times 1 \mathrm{~cm}^{2}$, on each side, is swept into the boundary. These considerations provide a flux balance for each source within the substrate:

$$
J_{y}(0, \bar{\delta})-2 J_{x}\left(\frac{1}{2} \delta, 0\right)=J_{y}(0,0) .
$$

At later times, the effective cross section of the lattice source may increase. This point and other details will be considered in a later paper. On reaching the surface, the flux vector $J_{y}\left(0, G_{T}\right)$ branches along two opposite directions along a surface slab of cross section $\delta^{\prime} \times 1$ $\mathrm{cm}^{2}$. For simplicity, it is assumed that $\delta=\delta^{\prime}$ giving the following coupling equation at the surface:

$$
J_{y}\left(0, G_{T}\right)=2 J_{x}\left(\frac{1}{2} \delta, G_{T}\right) \text {. }
$$

By applying Fick's first law, one obtains

$$
\frac{\partial C}{\partial y}-2 \frac{\partial C}{\partial x}=\mathscr{D}_{b} \frac{\partial C}{\partial y}
$$

for source coupling and

$$
\frac{1}{2} \mathscr{D}_{b} \frac{\partial C}{\partial y}=\mathscr{D}_{s} \frac{\partial C}{\partial x}
$$




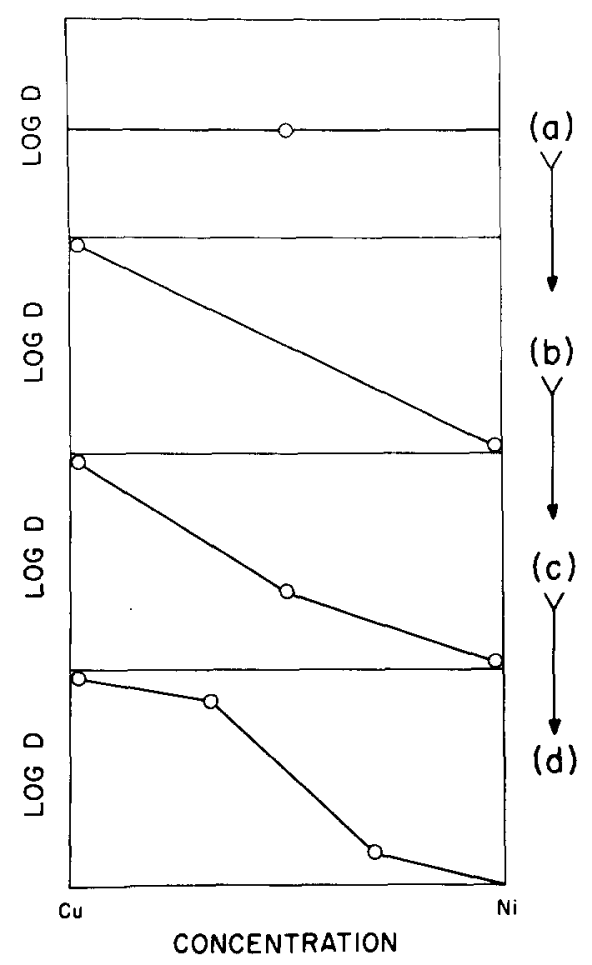

FIG. 7. System of plots useful for progressively refining the lattice diffusion coefficients to provide a fit between simulations and experimental data.

for surface coupling.

It is convenient to use the following dimensionless variables:

$$
\begin{aligned}
& x_{1}=x / \delta \mathscr{D}_{s}^{1 / 2}, \quad y_{1}=y / \delta \mathscr{D}_{b}^{1 / 2} \\
& D_{L}(C)=D(C) / D_{\max }, \quad t_{1}=D_{\max } t / \delta^{2},
\end{aligned}
$$

where $D_{\max }$ is the maximum value of $D(C)$. Substituting the new variables into $(6),(7),(9),\left(11^{\prime}\right)$, and (12'), gives

$$
\frac{\partial C}{\partial t_{1}}=\frac{1}{\mathscr{D}_{s}} \frac{\partial}{\partial x_{1}}\left(D_{L}(C) \frac{\partial C}{\partial x_{1}}\right)+\frac{1}{\mathscr{D}_{b}} \frac{\partial}{\partial y_{1}}\left(D_{L}(C) \frac{\partial C}{\partial y_{1}}\right)
$$

for the lattice;

$$
\frac{\partial C}{\partial t_{1}}=\frac{\partial}{\partial y_{1}}\left(D_{L}(C) \frac{\partial C}{\partial y_{1}}\right)+\frac{2 D_{L}(C)}{D_{S}^{1 / 2}} \frac{\partial C}{\partial x_{1}}
$$

for the grain boundary;

$$
\frac{\partial C}{\partial y_{1}}=\frac{\partial}{\partial x_{1}}\left(D_{L}(C) \frac{\partial C}{\partial x_{1}}\right)-\frac{D_{L}(C)}{\mathscr{D}_{b}^{1 / 2}} \frac{\partial C}{\partial y_{1}}
$$

for the surface;

$$
\frac{\partial C}{\partial y_{1}}-2\left(\frac{\mathscr{D}_{b}}{\mathscr{D}_{s}}\right)^{1 / 2} \frac{\partial C}{\partial x_{1}}=\mathscr{D}_{b} \frac{\partial C}{\partial y_{1}}
$$

for each substrate source; and

$$
\frac{1}{2} \mathscr{Q}_{b}^{1 / 2} \frac{\partial C}{\partial y_{1}}=\mathscr{Q}_{s}^{1 / 2} \frac{\partial C}{\partial x_{1}}
$$

for surface coupling.

\section{FINITE-DIFFERENCE SOLUTION TO DIFFUSION EQUATIONS}

For reasons already stated, no attempt will be made to obtain analytical solutions to Eqs. $\left(6^{\prime}\right),\left(7^{\prime}\right),\left(9^{\prime}\right),\left(11^{\prime \prime}\right)$, and $\left(12^{\prime \prime}\right)$. Instead, they will be solved in terms of finite differences in the nonlinear form. The spatial position of each point within a specific two-dimensional network is illustrated in Fig. 4, where

$$
\begin{array}{ll}
x_{1}=h_{x} j, & h_{x}=\Delta x / \delta \mathscr{D}_{s}^{1 / 2}, \\
y_{1}=h_{y} k, & h_{y}=\Delta y / \delta \mathscr{D}_{b}^{1 / 2} .
\end{array}
$$

For this special case, the source is located at $(j, k)$ $=(0,0)$, the surface coupling point is at $(0,5)$, the free surface is given by the set of points $(j, 5)$, and the original interface contains the points $(j, 0)$. Only one-half of a full network cell need be considered because of symmetry about the boundary points $(0, k)$. The composition points at $(4, k)$ are the mirror image of the points $(6, k)$ because of the symmetry plane at $(5, k)$. In the following discussion, the points $\left(j, k_{s}\right)$ will be used to locate the surface points for a general network.

Compositions at various times for the point $(j, k)$ are depicted by $C_{j k}^{+}, C_{j k}, C_{j k}^{-}$which correspond to $(n+1) \Delta t_{1}$, $n \Delta t_{1}$, and $(n-1) \Delta t_{1}$, respectively. Dimensionless time $\Delta t_{1}$ is related to real time by

$$
\Delta t_{1}=\left(D_{\max } / \delta^{2}\right) \Delta t
$$

If the dimensionless quantities are substituted into $\left(6^{\prime}\right)$, $\left(7^{\prime}\right),\left(9^{\prime}\right),\left(11^{\prime \prime}\right)$, and $\left(12^{\prime \prime}\right)$, one obtains the following difference equations:

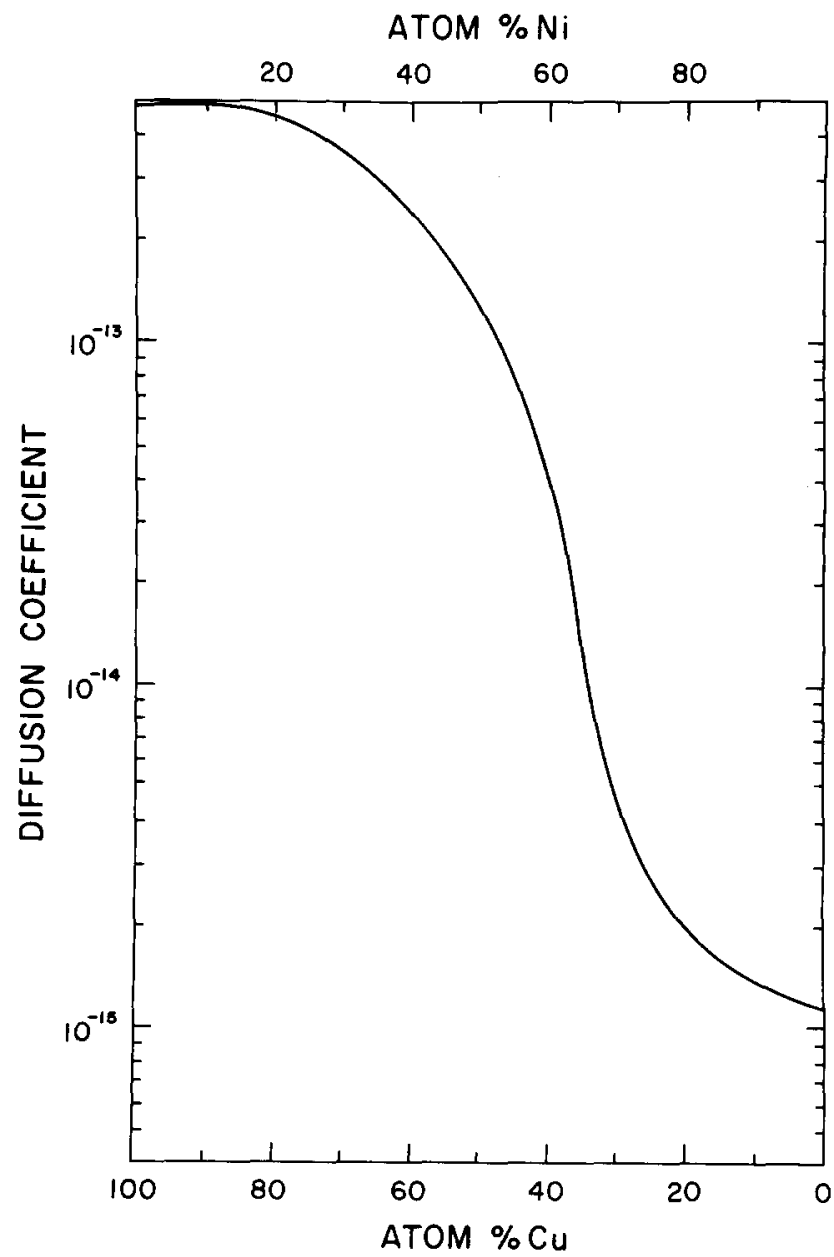

FIG. 8. Resultant volume diffusion coefficients $\left(\mathrm{cm}^{2} / \mathrm{sec}\right)$ for the $\mathrm{Cu}-\mathrm{Ni}$ system at $600^{\circ} \mathrm{C}$. 


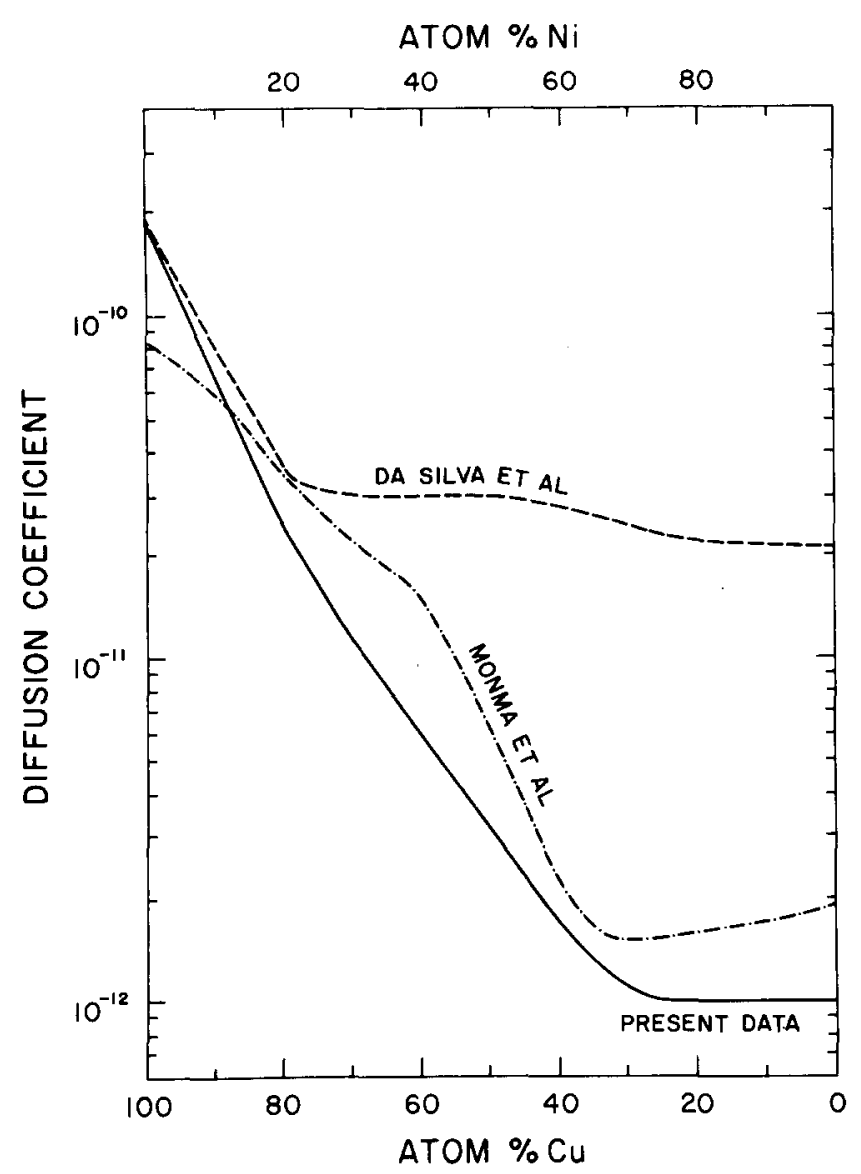

FIG. 9. Volume diffusion coefficients at $900^{\circ} \mathrm{C}$ obtained from $x$-ray simulations using the unified diffusion model (solid curve) as compared with other determinations.

$$
\begin{aligned}
C_{j k}^{+}= & C_{j k}+\left(\Delta t_{1} / h_{x}^{2} \mathscr{D}_{s}\right)\left(P_{j \pm 1, k}^{+} C_{j+1, k}-2 D_{j k} C_{j k}+P_{j \pm 1, k}^{-} C_{j-1, k}\right) \\
& +\left(\Delta t_{1} / h_{y}^{2} \mathscr{D}_{b}\right)\left(P_{j, k \pm 1}^{+} C_{j, k+1}-2 D_{j k} C_{j k}+P_{j, k \pm 1}^{-} C_{j, k-1}\right)
\end{aligned}
$$

for the lattice;

$$
\begin{aligned}
C_{0 k}^{+}= & C_{0 k}+\left(\Delta t_{1} / h_{y}^{2}\right)\left(P_{0, k \pm 1}^{+} C_{0, k+1}-2 D_{0 k} C_{0 k}+P_{0, k \pm 1}^{-} C_{0, k-1}\right) \\
& +2 \Delta l_{1}\left(D_{0 k} / \mathscr{D}_{s}^{1 / 2}\right)\left(C_{1 k}-C_{0 k}\right) / h_{x}
\end{aligned}
$$

for the grain boundary:

$$
\begin{aligned}
C_{j, k_{s}}^{+}= & C_{j, k_{s}}+\left(\Delta t_{1} / h_{x}^{2}\right)\left(P_{j \pm 1, k_{s}}^{+} C_{j+1, k_{s}}-2 D_{j k_{s}} C_{j, k_{s}}\right. \\
& \left.+P_{j \pm 1, k_{s}}^{-} C_{j-1, k_{s}}\right)-\Delta t_{1}\left(D_{j, k_{s}} / \mathscr{D}_{b}^{1 / 2}\right)\left(C_{j, k_{s}}-C_{j, k_{s}-1}\right) / h_{y}
\end{aligned}
$$

for the surface;

$$
\begin{aligned}
C_{0,0}= & C_{0,-1}+2\left(h_{y} / h_{x}\right)\left(\mathscr{D}_{b} / \mathscr{D}_{s}\right)^{1 / 2}\left(C_{1,0}-C_{0,0}\right) \\
& +\mathscr{D}_{b}\left(C_{0,1}-C_{0,0}\right)
\end{aligned}
$$

for substrate source; and

$$
C_{0, k_{s}}=\frac{C_{1, k_{s}}}{1+\frac{1}{2}\left(h_{x} / h_{y}\right)\left(\mathscr{D}_{b} / \mathscr{D}_{s}\right)^{1 / 2}}+\frac{C_{0, k_{s}-1}}{1+2\left(h_{y} / h_{x}\right)\left(\mathscr{D}_{s} / \mathscr{D}_{b}\right)^{1 / 2}}
$$

for surface coupling.

The coefficients are defined by

$$
P_{j \neq 1, k}^{+}=D_{j k}+\frac{1}{4}\left(D_{j+1, k}-D_{j-1, k}\right),
$$

$$
\begin{aligned}
& P_{j \pm 1, k}^{-}=D_{j k}-\frac{1}{4}\left(D_{j+1, k}-D_{j-1, k}\right), \\
& P_{j, k \pm 1}^{+}=D_{j k}+\frac{1}{4}\left(D_{j, k+1}-D_{j, k-1}\right), \\
& P_{j, k \pm 1}^{-}=D_{j k}-\frac{1}{4}\left(D_{j, k+1}-D_{j, k-1}\right) .
\end{aligned}
$$

Composition at the source point $(0,0)$ and coupling point $\left(0, k_{s}\right)$ may be obtained by applying $\left(11^{\prime \prime \prime}\right)$ and $\left(12^{\prime \prime}\right)$. Special equations are required because these points are not strictly a part of the lattice, boundary, or surface. Rather, they belong to combinations of these features. In the case of single-phase materials, all solutions are continuous.

\section{SIMULATION OF X-RAY INTENSITY BANDS}

The finite-difference solution of the diffusion equations may be used to generate $\mathrm{x}$-ray intensity bands which can be ultimately compared with experimental values. Generally, the first step in this computer calculation is to further refine the initial solution by an interpolation subroutine. Solution points are computer scanned for composition at each level below the surface. Each point of composition $m$ makes a contribution to an intensity band given by

$$
\Delta P_{m}=I_{0} Q_{m} \Delta V_{m e}^{g}(j k),
$$

where $\Delta V_{m e}^{g}(j k)$ is the incremental effective volume associated with the new rectangular grid. This may be expressed by

$$
\Delta V_{m e}^{g}(j k)=\left(2 / G_{L}\right) g A_{e} \Delta V_{m}(j k) \exp \left[-k_{m} \overline{\mu\left(Y_{k}\right)} Y_{k}\right],
$$

with $\Delta V_{m}(j k)$ representing the incremental volume ele ment within a half diffusion cell expressed in terms of

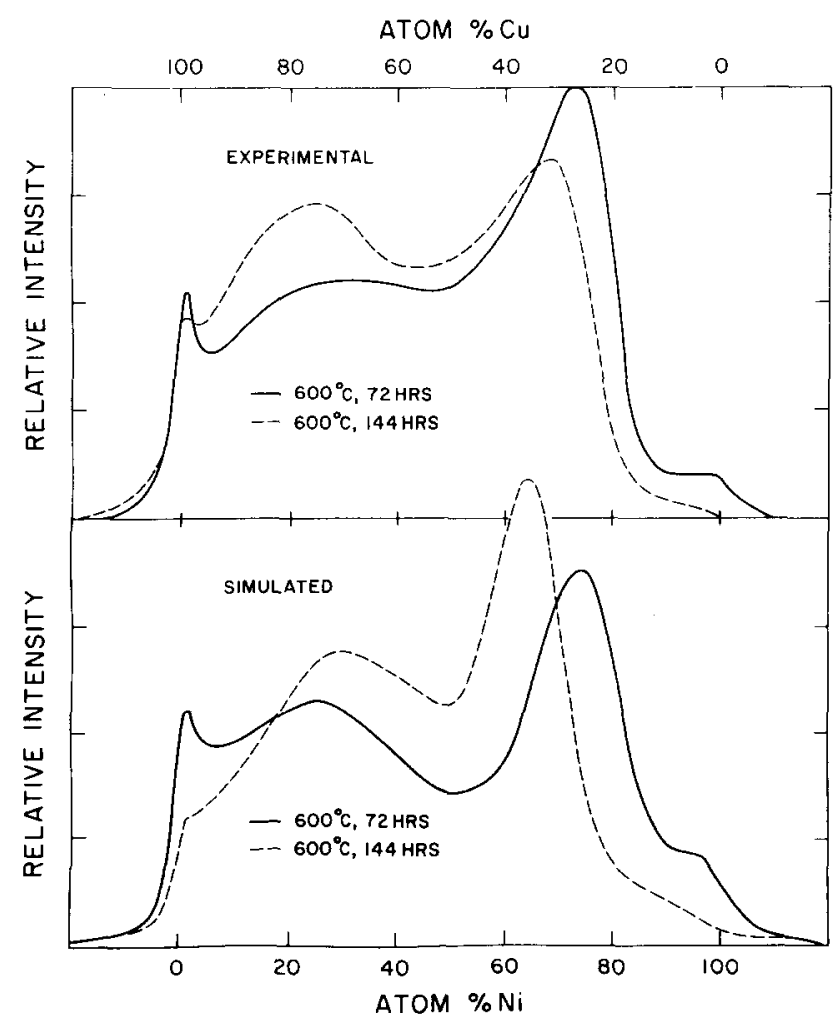

FIG. 10. Experimental and simulated intensities of the 111 intensity band for a specimen with $6.5 \mu$ of Ni electrodeposited onto a $\mathrm{Cu}$ single-crystal substrate. Diffusion times were 72 and $144 \mathrm{~h}$ at $600^{\circ} \mathrm{C}$. 


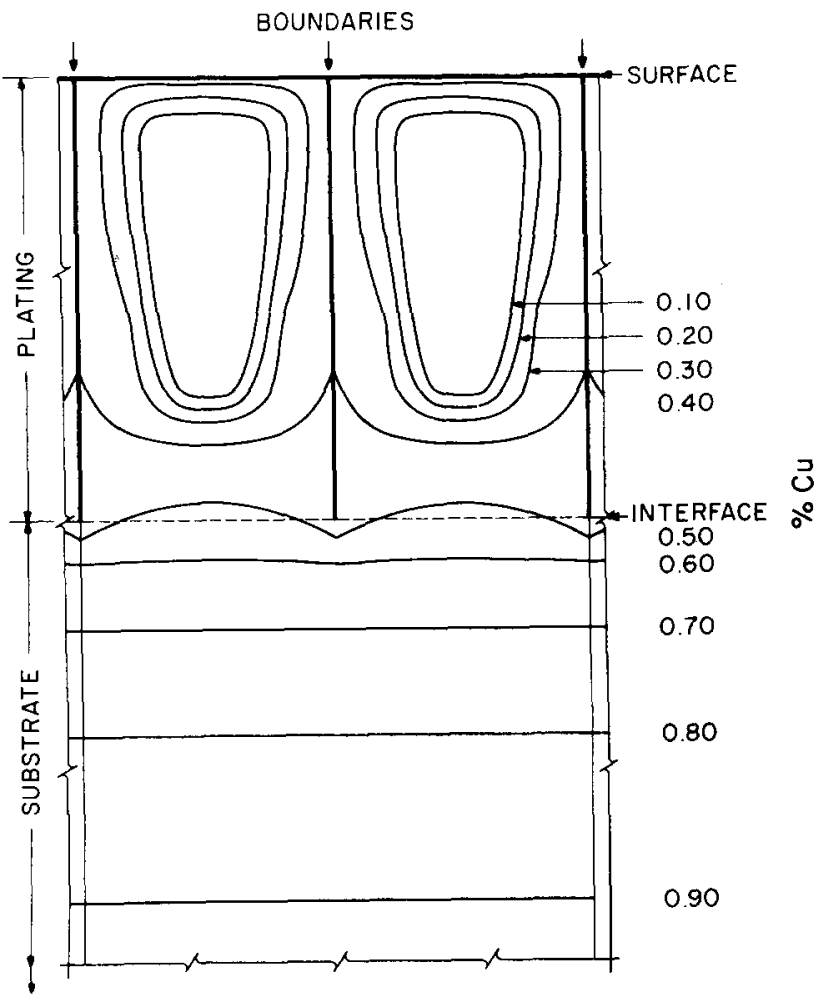

FIG. 11. Isoconcentration profiles for a grain size $1.8 \mu$, plating thickness $6.5 \mu$, and a diffusion treatment of $72 \mathrm{~h}$ at $600^{\circ} \mathrm{C}$. Two diffusion cells are illustrated for the $\mathrm{Cu}-\mathrm{Ni}$ system.

unit lengths out of the plane of Fig. $2, Y_{k}$ is the distance from the free surface (generally in $\mathrm{cm}$ ) and $\overline{\mu\left(Y_{k}\right)}$ is the average linear absorption coefficient $\left(\mathrm{cm}^{-1}\right)$ for all material from the surface to a thickness $Y_{k}$. For the $\mathrm{Cu}-\mathrm{Ni}$ system, a simple average may be used to approximate the absorption coefficient:

$$
\bar{\mu}=\frac{1}{2}\left(\mu_{\mathrm{Cu}}+\mu_{\mathrm{Ni}}\right) \text {. }
$$

This is valid only if the linear absorption coefficients are nearly equal.

The incremental effective volume element, $\Delta V_{m e}^{g}$, gives an intensity contribution over a $2 \theta$ range about some central value $\left(2 \theta_{m}\right)$ which varies with the composition $m$. The shape of this distribution is determined by the $\mathrm{x}$-ray geometry and the spectral broadening, and the integrated intensity by $\mathrm{Eq}$. $\left(4^{\prime}\right)$. It was found that a Cauchy instrumental broadening function provided a better fit to experimental data than a Gaussian ${ }^{10}$ and consequently was used for x-ray intensity band simulations. Each intensity band includes a system of overlapping instrumental profiles with one profile for each composition $m$. The number required to give smooth simulations depends upon the shape of the band and generally ranges from 30 to 50 .

\section{EXPERIMENTAL TECHNIQUES}

$\mathrm{X}$-ray diffraction data from $\mathrm{Cu}-\mathrm{Ni}$ samples diffused at $900^{\circ} \mathrm{C}$ have been described in detail elsewhere ${ }^{2,3}$ using a volume diffusion model. In this case, a conventional $\omega$ drive diffractometer was used with a $\beta$ filter which removed the Mo $K_{B}$ component, while the $K_{\alpha_{2}}$ component was eliminated with a modified Rachinger correction. Recently, a second arrangement, which removes the $K_{\alpha_{2}}$ experimentally, has been found to be preferable and was used for collecting the $600^{\circ} \mathrm{C}$ data presented here. This required a singly bent and symmetrically ground incident beam quartz monochromator and a fine-focus $\mathrm{Cu}$ diffraction tube. No soller slits are used since a large axial divergence (about $\pm 4^{\circ}$ ) is needed to permit crystals misoriented along the $\chi$ axis to diffract. The axial divergence should be made as large as the maximum width of the rocking curve. Also, a receiver slit was selected which was slightly larger than the tube focal spot viewed at a $6^{\circ}$ take-off angle. The $\lambda / n$ components of radiation were removed by using a scintillation counter with a pulse-height analyzer. All x-ray components were supplied by Siemens with the exception of a Stoe Eulerian cradle. Each rocking curve was recorded at $\frac{1}{4} \mathrm{deg} / \mathrm{min}$ and the integrated intensity at each $2 \theta$ position was taken as the total area under each curve. The width of each rocking curve was characterized by its variance. This approach provides a simple means of correcting for instrumental broadening since the measured variance is the sum of the variance of a Gaussian misorientation function and the instrumental function. ${ }^{3}$ The corrected variances representing only misorientation are given in Fig. 5 .

All $\mathrm{Cu}$ single crystals were oriented and cut from a $\frac{3}{4}-$ in. -diam rod to a thickness of about $\frac{1}{4}$ in. The crystals were of $99.99 \%$ purity, had a dislocation density of about $10^{5} / \mathrm{cm}^{2}$, and were supplied by Research Crystals Inc. After acid polishing, the orientation was rechecked and in each case a (111) plane was within $1^{\circ}$ of the surface. Optical examination of the surface indicates that irregularities were less than $0.37 \mu$ in height.

Two specimens are considered. One contained an $8-\mu$ electrodeposit of $\mathrm{Ni}$ and was diffused at $900^{\circ} \mathrm{C}$ while 6.5 $\mu$ was used for a specimen diffused at $600^{\circ} \mathrm{C}$. Thicknesses were determined from weight gain and $\mathrm{x}$-ray absorption measurements. A grain size distribution is required for those grains within the diffusion zone before meaningful computer simulations can be carried out using the unified diffusion model presented in Sec. III. Such a distribution can be related to $G_{L}$ and $n G_{L}$. Scanning electron and optical microscope pictures were obtained before and after systematic etching of surface

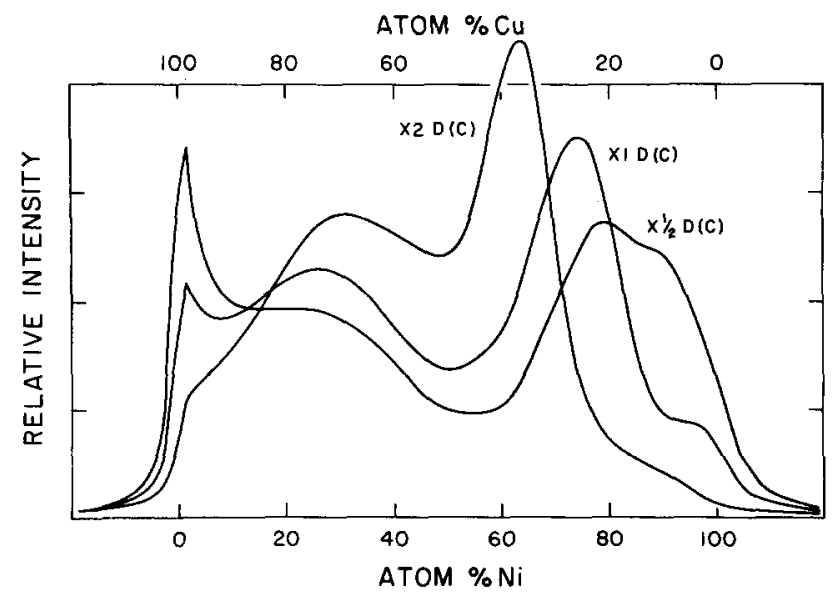

FIG. 12. Response of the $111 \mathrm{x}$-ray intensity band to changes in $D(C)$ by factors of 2 and $\frac{1}{2}$. The simulations refer to a $\mathrm{Cu}-\mathrm{Ni}$ sample diffused for $72 \mathrm{~h}$ at $600^{\circ} \mathrm{C}$ containing $6.5 \mu$ of Ni. Also, the bimodal grain size distribution given in Fig. 6 was used. 


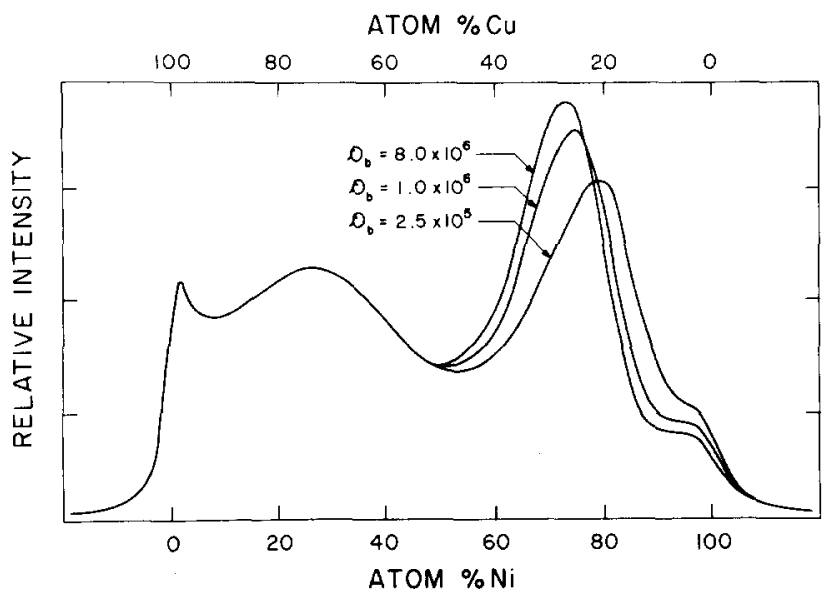

FIG. 13. All conditions were the same as for Fig. 12 except $\mathscr{D}_{b}$ was varied by factors of 8 and $\frac{1}{4}$. Also, $\times 1 D(C)$ was used for all simulations.

material in order to obtain a grain size distribution, as well as information concerning the variation of such a distribution with distance. In order to preserve a specimen for continued diffusion treatments, a sample was examined by etching only after the last anneal. No obvious change was observed in the grain size distribution with distance, provided the electrodeposited region was under examination. However, a large size change was observed when the electrodeposited region was totally removed leaving only material which was originally pure copper substrate. This suggests that the spacing between boundaries, $n G_{L}$, within the substrate, is sufficiently large to be neglected for the present samples. Consequently, only the size distribution within the plating need be considered and this appears to be independent of distance below the surface. The distribution after the $600^{\circ} \mathrm{C}$ diffusion treatment spans a range extending from 0.2 to $2.2 \mu$ and is given in Fig. 6. It is important to note that the diffracted intensity is proportional to the number of unit cells; therefore, the distribution has been weighted to give the relative volume associated with each size range. Figure 6 illustrates that most of the specimen volume consists of grains close to $1 \mu$. Consequently, preliminary simulations based upon the unified model for $600^{\circ} \mathrm{C}$ were obtained with $G_{L}$ at or near $1.0 \mu$.

\section{COMPUTER SIMULATIONS}

All quantities except $D(C), D_{b}$, and $D_{s}$ may be determined directly from supporting measurements or are defined by convention $\left(\delta=5 \times 10^{-8} \mathrm{~cm}\right)$. The diffusion coefficients, or their ratios, may be obtained by adjusting their values until a fit is obtained with the $\mathrm{x}$-ray diffraction data. Fortunately, the detailed response of the model to parameter adjustment is sufficiently unique for each of the quantities to be determined separately. Considerable computer time may be saved if good initial estimates are found for the diffusion coefficients. The following starting relations ${ }^{11}$ were found to be useful between activation energies and frequency factors for volume, grain boundary, and surface diffusion:

$$
\begin{aligned}
& Q=2 Q_{b}=4 Q_{s}, \\
& D_{o}=2 D_{o b}=4 D_{o s},
\end{aligned}
$$

along with the well-known melting-point relationship

$$
Q=38 T_{\mathrm{MP}}
$$

for fcc materials.

An estimate of the frequency factor may be obtained from the Dushman-Langmuir equation

$$
D=D_{o} \exp (-Q / R T),
$$

where

$$
D_{o}=Q a^{2} / N h=1.04 \times 10^{-3} Q a^{2}
$$

with $a$ being a lattice constant, $N$ Avagadro's number, and $h$ Planck's constant. Other useful forms may be found in the literature. ${ }^{11}$

It was discovered, for the present problem, that the surface composition is insensitive to reasonable variations of $D_{s}$ about the starting values given by (22) and (23) and a further simplification could be obtained by assuming that the surface composition is independent of $x$. This results from large values for $D_{s}$ and small $G_{L}$. If $G_{L}$ is relatively large, it is likely that a significant concentration gradient could develop along the surface and would have to be considered to obtain accurate solutions. This simplification reduces the number of unknowns to the curve $D(C)$ and the ratio $\mathscr{D}_{b}$.

Although a study of the behavior of one alloy system may not be typical for other systems with complete solid solubility, certain generalities might be expected. Scaling $D(C)$ by adjusting $D_{\max }$ provides a selection of the time scale, i.e., if a considerable spread of $x$-ray intensities is observed and only a small amount of the pure
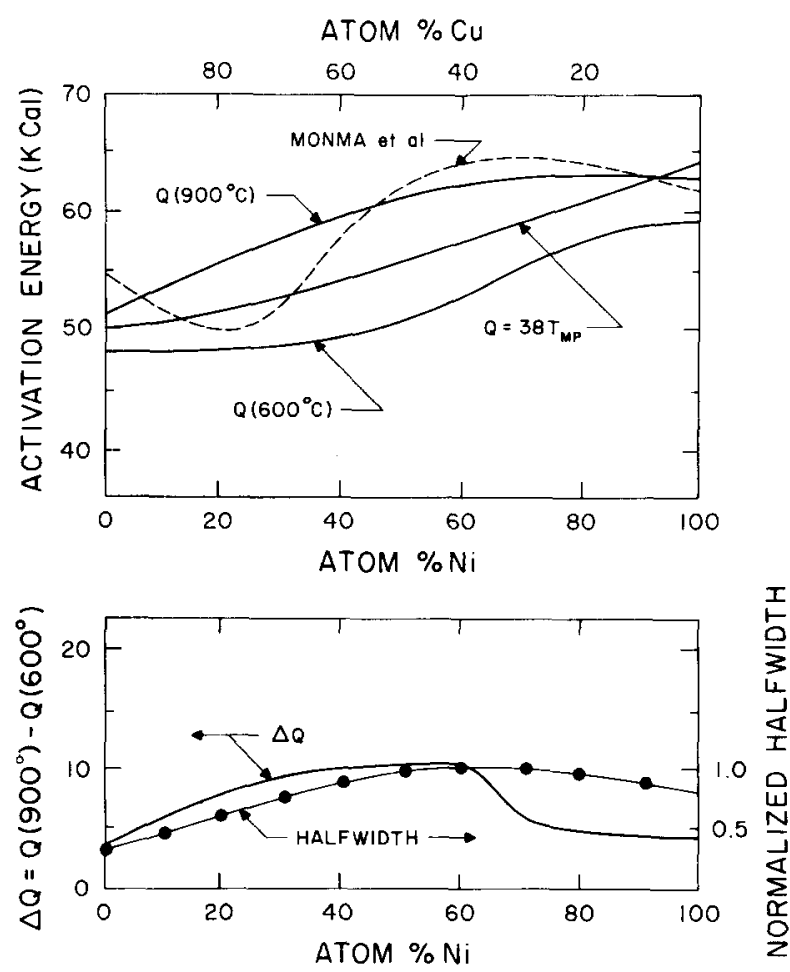

FIG. 14. Upper curve: activation energies obtained by the x-ray approach for $900^{\circ} \mathrm{C}$ and $600^{\circ} \mathrm{C}$ data, compared with Monma $e t a l$. and the melting-point relationship. Lower curve: difference in $900^{\circ} \mathrm{C}$ and $600^{\circ} \mathrm{C}$ activation energies, $\Delta Q$, compared with normalized half-widths from rocking curves. 
starting materials remains, then $D_{\max }$ may be adjusted to generally describe the extent of diffusion within the penetration range of the $\mathrm{x}$-ray beam. On the other hand, if the simulations indicate greater intensity-band formation than that observed, the value of $D_{\max }$ should be reduced.

The shape of the $D(C)$ curve greatly influences the shape of the x-ray intensity bands. Consequently, the major effort in parameter adjustment is likely to result from systematic variations of the shape of the lattice diffusion coefficient curve. It has been suggested by Wagner ${ }^{12}$ that a logarithmic form fits much of the diffusion coefficient data for binary metallic systems. A curve defined by a system of connecting straight lines was found to be useful in refining the computer simulations [see Figs. $7(\mathrm{a})-7(\mathrm{~d})]$. This might begin with a straight line and continue to two, three, or more segments until a suitable fit is obtained with the x-ray data. A smooth curve without slope discontinuities should be constructed as a final refinement of $D(C)$ for a single phase (Fig. 8).

It is evident that $\mathscr{D}_{b}$ must also be adjusted about some reasonable starting point. An adjustment of this parameter primarily influences the composition contours within the plated material, especially, the composition of the grain boundary and free surface. This parameter is therefore influential over a limited composition range and it is this distinction that makes an independent evaluation possible. The influence of variations in $\mathscr{D}_{b}$ should be determined for each curve $D(C)$.

It was discovered, after a response study, that certain features of intensity bands on the nickel side could not be described by simulations using a single grain size. After obtaining a best fit for diffusion parameters by using an average grain size, the model was further tested by simulating intensity bands having the range of grain sizes determined by experiment (Fig. 6). It was discovered that a small intensity peak, relatable to pure nickel and observed after $72 \mathrm{~h}$ at $600^{\circ} \mathrm{C}$, is found in simulations obtained from the largest observed grain sizes. However, most other features are better described if a small grain size parameter is used. This leads one to the conclusion that the over-all grain size distribution can be an important consideration, and an attempt was made to include various sizes into the analysis. A simple bimodal distribution consisting of $0.6 \mu$ (weighted by $\frac{2}{3}$ ) and a larger size of $1.8 \mu$ (weighted by $\frac{1}{3}$ ) describes all of the features of the $\mathrm{x}$-ray data and is compatible with the observed distribution.

Figures 8 and 9 illustrate the $D(C)$ curves obtained for 900 and $600^{\circ} \mathrm{C}$. The $900^{\circ} \mathrm{C}$ diffusion data were obtained after visual fitting of the 222 and 333 intensity bands at $\frac{3}{4}, 2$, and $5 \mathrm{~h}$. Figures $10(\mathrm{a})$ and $10(\mathrm{~b})$ illustrate experimental results and simulations for the 111 intensity bands after diffusion treatments of 72 and $144 \mathrm{~h}$ at $600^{\circ} \mathrm{C}$. An additional simulation check was made using the experimental 222 intensity bands obtained at the same temperature and times. The values of $\mathscr{D}_{b}\left(900^{\circ} \mathrm{C}\right)$ and $\mathscr{D}_{b}\left(600^{\circ} \mathrm{C}\right)$ were found to be $1 \times 10^{4}$ and $1 \times 10^{6}$, respectively.

One can relate the major features of Fig. 10 (a) $\left(600^{\circ} \mathrm{C}\right.$, $72 \mathrm{~h}$ ) to a specific range of compositions. For example, the intensity peak at $30 \% \mathrm{Cu}-70 \% \mathrm{Ni}$ can be related to material along the grain boundary and surface. There is also a zone at about $4 \mu$ below the surface with this as an average composition (refer to Fig. 11). All of these regions combine to give a large effective volume and therefore a large $\mathrm{x}$-ray intensity near this composition. At a distance of $2 \mu$ a pocket of nearly pure $\mathrm{Ni}$ continues to be found after $72 \mathrm{~h}$. It is interesting to note that between 0 and $4 \mu$ the boundary acts as a source of $\mathrm{Cu}$ atoms, while from $4 \mu$ to the interface the boundary acts as a sink. This behavior was first predicted by Suzuoka ${ }^{9}$ using a more approximate model; however, further details will be presented in a future paper.

Figure 12 illustrates that large changes are found in computer simulations of the $\mathrm{x}$-ray intensity bands after $D(C)$ is rescaled by factors of 2 and $\frac{1}{2}$. Significant changes were also observed after making slight adjustments in the shape of the $D(C)$ curve. This response suggests that the $x$-ray method provides a high degree of accuracy in the determination of $D(C)$. The response to changes in $\mathscr{D}_{b}$ is illustrated in Fig. 13 and it can be seen that only compositions in excess of $50 \% \mathrm{Ni}$ are affected. Also, the over-all intensity change is smaller if $\mathscr{D}_{b}$ is varied as compared with a similar variation in $D(C)$. In the latter case, the entire diffusion zone is influenced while variations in $\mathscr{D}_{b}$ directly influence only material containing boundaries. The response of the intensity simulations with variations in $D(C)$ and $\mathscr{D}_{b}$ is sufficiently different to permit each to be determined independently.

\section{RESULTS AND CONCLUSIONS}

Sufficient data are available for the $\mathrm{Cu}-\mathrm{Ni}$ system to make it a good choice for checking results from equations presented in previous sections. Some results are given in Figs. 8 and 9 along with other independent determinations. Equation (27) provides a relationship giving $D_{0}$ and $Q$ from diffusion coefficient data at a single temperature. For the present data, Fig. 14, the fre quency factor can be taken as 0.7 and independent of composition. It can be seen that the best agreement is found with the most recent data obtained by Monma et al. ${ }^{13}$ using radioactive tracers. In another paper, ${ }^{14}$ one of the authors presented pseudo-diffusion coefficients for the $\mathrm{Cu}-\mathrm{Ni}$ system at $900^{\circ} \mathrm{C}$. These were obtained using simulations based entirely upon a volume diffusion model. The coefficients on the nickel-rich side were intermediate between those reported by DaSilva and $\mathrm{Mehl}^{15}$ and Monma et al. ${ }^{13}$ At this point, it is clear that high values for the diffusion coefficient will be obtained at $900^{\circ} \mathrm{C}$ from polycrystalline $\mathrm{Cu}-\mathrm{Ni}$ specimens unless a correction is made for grain size. This problem was greatly reduced by Monma by using large-grain specimens and by diffusing a series of alloys just below the solidus. For the present work, the ratio of diffusion temperature $\left(1173^{\circ} \mathrm{K}\right)$ to the solidus gives a range from 0.78 to 0.68 for compositions between 50 and $100 \% \mathrm{Ni}$. Although it is convenient to have a rule which provides a practical temperature range over which pure lattice diffusion is dominant, this must be treated with considerable caution ${ }^{16-18}$ since the lower limit is grain size dependent and depends upon the accuracy of the method.

In the Appendix an Arhennius form is suggested for $\mathscr{D}_{b}$ which may be written as 


$$
\mathscr{D}_{b}=0.015 \mathrm{exp}\left(-31 \times 10^{3} / R T\right)
$$

based upon measurements at 900 and $600{ }^{\circ} \mathrm{C}$. Since $D_{b}$ $=\mathscr{D}_{b} D(C)$, the following form may be more useful:

$$
D_{b}(C)=0.0105 \exp \left\{-[Q(C)-31] \times 10^{3} / R T\right\}
$$

and may be compared with several sets of data already in the literature. Results are available for grain boundary diffusion of $\mathrm{Ni}$ into $\mathrm{Cu}{ }^{19}$ which make use of bicrystals having a range of angles. It was found that the activation energy for grain boundary diffusion decreases from a value associated with pure lattice diffusion to about $38 \mathrm{kcal}$ and remains constant between the angles $30^{\circ}$ and $60^{\circ}$. The decrease below the activation energy for lattice diffusion is $26 \mathrm{kcal}$.

Another set of data is available using the same technique for grain boundary self-diffusion in $\mathrm{Ni} .{ }^{20}$ In this case, a similar change was observed with grain boundary angle, i.e., for very low angles the grain boundary activation energy approaches the lattice activation energy and for higher angles it becomes nearly constant at $26 \mathrm{kcal}$ in the range from $20^{\circ}$ to $70^{\circ}$. This represents a reduction of about $40 \mathrm{kcal}$ below the lattice activation energy reported by these authors. If this is compared with the value 26 , obtained for $\mathrm{Ni}$ diffusing into $\mathrm{Cu}$, one obtains an average reduction of $33 \mathrm{kcal}$, which might be compared with $31 \mathrm{kcal}$, the value obtained in the present investigation. Also, a value of $28.2 \pm 2.0 \mathrm{kcal}$ has been reported for the grain boundary activation energy for self-diffusion in polycrystalline $\mathrm{Ni}^{21}$ together with a frequency factor of

$$
\begin{aligned}
& 1.75^{-1.0}+2.1 \\
& +10^{-2}
\end{aligned}
$$

This comes close to the present value of $32 \mathrm{kcal}$ for pure $\mathrm{Ni}$ and a frequency factor of $1.05 \times 10^{-2}$. However, it should be noted that this study is based upon boundaries in $\mathrm{Cu}-\mathrm{Ni}$ alloys ranging in composition from about $50 \%$ $\mathrm{Ni}$ to 100 at. \% Ni. Also, they were formed by electrodeposition of $\mathrm{Ni}$ on $\mathrm{Cu}$ single crystals. Boundaries formed in this way may not be like those obtained using bicrystals or like polycrystalline boundaries.

A reexamination of Fig. 14 is in order at this point since there is a significant discrepancy in activation energies obtained from 900 and $600^{\circ} \mathrm{C}$ data. The activation energy for volume diffusion at $600^{\circ} \mathrm{C}$ is up to $\frac{1}{6}$ lower than that obtained at $900^{\circ} \mathrm{C}$ and this cannot be explained by experimental error. The lower portion of Fig. 14 includes a plot of $Q\left(900^{\circ} \mathrm{C}\right)-Q\left(600^{\circ} \mathrm{C}\right)=\Delta Q$ and has a maximum between 40 and $60 \% \mathrm{Ni}$. In the same portion of this figure, one finds a plot of normalized half-width, calculated from the variance curves of Fig. 5. The two curves have the same form up to $60 \% \mathrm{Ni}$. Deviations above $60 \%$ probably result from the close proximity of the free surface which requires special considerations. A correlation between $\Delta Q$ and the rocking curve halfwidth is reasonable if the crystal misorientation results from additional dislocations lines extending through the lattice in the form of three-dimensional arrays. Dislocations acting as high-diffusivity pipes are expected to be especially important at $600^{\circ} \mathrm{C}$, a relatively low diffusion temperature. If $600^{\circ} \mathrm{C}$ is compared with the solidus temperatures for $\mathrm{Cu}-50$ to $100 \% \mathrm{Ni}$, ratios be- tween 0.59 and 0.50 are obtained. This is a transition range where pipe diffusion may begin to make a more significant contribution than pure lattice diffusion. Although the activation energy for volume diffusion has been lowered by $\frac{1}{6}$ relative to that obtained at $900^{\circ} \mathrm{C}$, it is still well above the activation energy for grain boundary diffusion since this is approximately $\frac{1}{2}$ the energy associated with lattice diffusion. Such a difference is still sufficient to treat the diffusion problem in terms of two materials, i.e., grain boundaries and a threedimensional volume with an enhanced pseudo-diffusion coefficient. At lower temperatures, this picture may not be valid and the present unified model may not be applicable.

\section{ACKNOWLEDGMENTS}

The authors are grateful to the U.S. Army Research Office-Durham and the National Science Foundation for funding this research.

\section{APPENDIX: FORM OF GRAIN BOUNDARY DIFFUSION COEFFICIENT}

The form of the grain boundary diffusion coefficient deserves further discussion since other relations between the frequency factors and activation energies must also exist. In taking $D_{b}=\mathscr{D}_{b} D(C)$, with $\mathscr{D}_{b}$ independent of composition, one assumes that the chemical environment influences atomic diffusion along the grain boundary in the same way it does for atomic diffusion through the lattice. In other words, a high (or low) atomic diffusion rate through a lattice of composition $C$ implies that a comparable high (or low) diffusion rate will be found along a grain boundary of the same composition, provided the same driving force acts for both cases. The coefficient $\mathscr{D}_{b}$ merely allows for the much higher diffusivity resulting from the structural rearrangement associated with boundary material.

If conventional Arhennius forms are taken for both diffusion coefficients, the ratio of coefficients $\mathscr{D}_{b}$ is given by $\mathscr{D}_{o b} \exp (\Delta Q)$, with $\Delta Q=Q-Q_{b}$, the difference between the lattice and boundary activation energies. Since it has been assumed that $\mathscr{D}_{b}$ is independent of composition, $\Delta Q$ and $\mathscr{D}_{o b}\left(=D_{o b} / D_{o}\right)$ are also taken to be independent of composition, and relatable to the structural rearrangement of high-diffusivity grain boundaries. If the lattice frequency factor varies with composition, it is assumed that the boundary frequency factor has the same functional dependence.

The parameter $\mathscr{D}_{b}$ may be obtained by trial and error at several temperatures using the unified model described in the text. This enables both $\mathscr{D}_{o b}$ and $\Delta Q$ to be evaluated.

\footnotetext{
*Present address: Chrysler Corp. Box 1118, CIMS 416-30-03, Detroit, Mich. 48231.

${ }^{1}$ C. R. Houska, J. Appl. Phys. 41, 69 (1970).

${ }^{2}$ D. R. Tenney, J. A. Carpenter, and C. R. Houska, J. Appl. Phys 41, 4485 (1970)

${ }^{3}$ J. A. Carpenter, D. R. Tenney, and C. R. Houska, J. Appl. Phys. 42, 4305 (1971).

${ }^{4}$ E. W. Hart, Acta Metall. 5, 597 (1957).

${ }^{5}$ J. C. Fisher, J. Appl. Phys. 22, 74 (1951).

${ }^{6}$ This conclusion is based upon unpublished computer simulations carried out by D. R. Tenney.
} 
${ }^{7}$ R. T. P. Whipple, Philos. Mag. 45, 1225 (1954)

${ }^{8}$ H. S. Levine and C. J. MarCallurn, J. Appl. Phys. 31, 595 (1960).

${ }^{9}$ T. Suzuoka, Nippon Kinzoku Gakkai, Trans. Japan Inst. Metals 2, 25 (1961).

${ }^{0}$ E. R. Pike, Acta Crystallogr. 12, 87 (1959)

${ }^{11}$ J. Askill, Tracer Diffusion Data for Metals, Alloys, and Simple Oxides (Plenum, New York, 1970).

${ }^{12}$ C. Wagner, J. Met. 4, 91 (1952).

${ }^{13} \mathrm{~K}$. Monma, H. Suto, and H. Okiawa, Nippon Kinaoku Gakkhaishi 28, 192 (1964).

${ }^{14} \mathrm{C}$. R. Houska, in Third European Conference on Thermophysical Properties at High Temperature, Torino, Italy, High Temp.-High Pressures (to be published).

${ }^{15}$ L. C. DaSilva and R. F. Mehl, Trans. AIME (Am. Inst. Min. Metall.
Pet. Eng.) 191, 155 (1951).

${ }^{16}$ There appears to be no well-agreed-upon range over which lattice diffusion truly predominates. A range of $2 / 3<T / T_{\mathrm{MP}}<1$ has been suggested by one author (Ref. 17) while another (Ref. 18) indicates a lower limit of $3 / 4$. In this work, a value of $4 / 5$ would be preferred.

${ }^{17} \mathrm{P}$. G. Shewman, Diffusion in Solids (McGraw-Hill, New York, 1963).

${ }^{18} \mathrm{~J}$. W. Christian, The Theory of Transformations in Metals and Alloys (Pergamon, New York, 1965)

${ }^{19} \mathrm{~S}$. Yukawa and M. J. Sinnot, Trans. AIME (Am. Inst. Min. Metall. Pet. Eng.) 203, 996 (1955).

${ }^{20}$ W. R. Upthegrove and M. J. Sinnott, ASM Trans. Q. (Am. Soc. Met.) 50, 1031 (1958)

${ }^{21}$ W. Lange, A. Hassner and G. Mischer, Phys. Status Solidi 5, 63 (1964). 